Indexed by

\title{
PROPOSED MODELS TO CALCULATE AND OPTIMISE LINE CAPACITY UNDER DIFFERENT OPERATION CONDITIONS FOR EGYPTIAN RAILWAY NETWORK
}

Diana M. S. Rahoma

Ain Shams University,

Faculty of Engineering,

Public Works Department,

Cairo, Egypt

Akram S. Kotb

Building and Construction

Department. College of

Engineering and Technology,

Arab Academy for Science

and Technology and

Maritime Transport,

Cairo, Egypt

\author{
Ali Z. Heikal \\ Ain Shams University, \\ Faculty of Engineering, \\ Public Works Department, \\ Cairo, Egypt
}

\author{
Haytham N. Zohny \\ Ain Shams University, \\ Faculty of Engineering, \\ Public Works Department, \\ Cairo, Egypt
}

Key words: official capacity, capacity calculation, capacity factors, regression analysis, analytical method, official time table, line capacity

\section{Cite article:}

Diana, M. S. R., Ali, Z. H., Haytham, N. Z., \& Akram, K. S. [2021]. Proposed models to calculate and optimise line capacity under different operation conditions for egyptian railway network. Journal of Applied Engineering Science, 19(1), 77 - 83. DOl:10.5937/jaes0-27821

Online aceess of full paper is available at: www.engineeringscience.rs/browse-issues 


\title{
PROPOSED MODELS TO CALCULATE AND OPTIMISE LINE CAPACITY UNDER DIFFERENT OPERATION CONDITIONS FOR EGYPTIAN RAILWAY NETWORK
}

\author{
Diana M. S. Rahoma ${ }^{1 *}$, Ali Z. Heikal', Haytham N. Zohny', Akram S. Kotb ${ }^{2}$ \\ ${ }^{1}$ Public Works Department, Faculty of Engineering, Ain Shams University, Cairo, Egypt \\ ${ }^{2}$ Building and Construction Department. College of Engineering and Technology, Arab Academy for \\ Science and Technology and Maritime Transport, Cairo, Egypt
}

The capacity evaluation models are planning tools that help government agencies to maximize the benefit from existing railway infrastructure and improve rail transportation operations. These models determine the maximum number of trains that could operate on a given railway infrastructure, during a specific time interval under operational conditions. This paper develops analytical models to calculate the railway line capacity using a regression analysis based on the Egyptian official timetable. The Egyptian railway network consists of 104 links under different operation conditions (passenger / freight, passenger and freight trains) with mechanical or electrical signals systems running on single or double tracks. To calculate railway practical capacity, the three equations for each operation conditions were improved by the combination of the longest block section and passenger and freight speed. These equations have accepted value of the coefficient of determination and the absolute average error. Finally the maximum capacity is obtained by the optimum values of effective factors using iteration technique. This optimum values will increased about $80 \%$ of the capacity for Egyptian network lines.

Key words: official capacity, capacity calculation, capacity factors, regression analysis, analytical method, official time table, line capacity

\section{INTRODUCTION}

Railway capacity analysis is the most significant concern of railway operators that needs an extensive planning and optimizing the use of limited resources. The evaluation of railway line capacity is a complex problem that depends on a combination of influencing factors [1].

UIC is defined the capacity as the maximum number of trains traversing a specific part of the rail during a given time period at a fixed level of service [2]. Railway capacity is classified into theoretical and practical capacity. Theoretical capacity is the number of train runs over a line per day under ideal conditions which is easy to generate and useful for long term strategic goals. While practical capacity is the practical limit of actual traffic volume that can be moved on a line under defined performance which is estimated as $75 \%$ of the theoretical capacity because of real world limitations. It is important for operational stages [3], [4].

Several studies proposed different methods to evaluate railway capacity. Pachl (2018) classified the capacity methodologies into two types only: analytical and simulation [5]. Abril et al. (2008) divided the methods into three categories: analytical, optimization, and simulation methods [6]. But Han (2016) classified these methods into four methods: Analytical, Graphical, Optimization, and Simulation Methods [7].

The analytical railway capacity models used to evaluate railway capacity by developing a set of mathematical relations that allow railway planners to measure the performance of railways. Many researches measure and improve the railway capacity using analytical models as:

- UIC 405 (1996) presented the delays as important factor affected on capacity analysis. The capacity evaluation is based on operating plan represented on timetables while UIC406 (2004) developed a methodology to evaluate railway capacity by compressing the existing timetable and calculate the infrastructure occupation time. The capacity consumption was achieved by adding the buffer time to the occupation time [2].

- Ali (2012) was noted that the highest speed leads to the highest track capacity and presented an analytical study for the effect of high speed in developing and emerging countries [8].

- Connor (2014) determined line capacity as it depended on train performance, particularly braking and acceleration, length and how trains are controlled at high speed lines [9].

- Lindfeldt (2015) and Huelsz (2015) studied the effect of configuration of the timetable on capacity. Capacity estimation mainly depends on the train operation according to a planned timetable as they only can meet or pass each other at discrete locations $[4,10]$.

- Sangphong et al (2016) presented a model to maximize rail line capacity by minimizing the train headway. This analysis was conducted under a fixed-block system, which allows only a single train to remain in the block. The maximum capacity was achieved 
when two trains operated at the same speed [11].

- Zhong (2019) presented an analytical tool to evaluate railway capacity based on blocking time theory. It concerned that the timetabling data was remain constant during scheduling. This theory depended on the critical block section for describing the capacity utilization [12].

Capacity calculation for the previous studies depend on only one factor as delay time, speed, train head way and blocking time.

In this paper, analytical models were developed to calculate and improve railway capacity taken into consideration the combination between the most effective factors otherwise the previous studies. The used data in the analysis are based on Egyptian national railway timetable.

\section{METHODOLOGY \& DATA COLLECTION}

The objectives were divided into two stages:

First stage: Creating mathematical models for predicting railway practical capacity using several influencing factors and based on the Egyptian national railway (ENR) official time table by regression software Statistical Package for the Social Sciences (SPSS).

For evaluating the model, certain measures are used to assess the model's goodness-of-fit to the data as:

- The correlation coefficient: the relationship exists between two variables, it explains how strong that relationship is and whether the relationship is positive or negative [13].

- The coefficient of determination $\left(\mathrm{R}^{2}\right)$ : the variation in one variable is directly related to variation in another variable. It must be bounded by 0 and 1 [13]. $R^{2}=1$ is a 'perfect score', obtained only if the data points happen to lie exactly along a straight line; $R^{2}=0$ is perfectly lousy score

- The sign of factors (+and -)

- The significance of model coefficients.

- The standard error of estimate: the accuracy of the predictions.

Second stage: Calculating the optimum value for factors to maximize the railway capacity using an iteration technique by solver program. The iteration is a mathematical way of solving a problem which generates a sequence of approximations to reach the maximum values. This method is applicable for both linear and nonlinear problems with large number of variables [14].

Calculating capacity is a complicated process as it depends on numerous infrastructure and operational factors. The most affecting factors were taken as input data such as the longest block section (Lb) km, signal system (S.S), average speed for passenger and freight (Sp and Sf) kph, track type (T.T), number of blocks (Nb), number of level crossing (L.C), passenger/ $Q_{\text {official }}(P / Q)$ and freight $/ Q_{\text {official }}(F / Q)[15]$. These factors were based on previous studies; experiences and field survey are shown in table 1.

Table 1: The important factors, the official capacity $\left(Q_{\text {official }}\right)$ of links

\begin{tabular}{|c|c|c|c|c|c|c|c|c|c|c|}
\hline Link Name & $\mathrm{Nb}$ & $\mathrm{Lb}$ & $\mathrm{T} . \mathrm{T}$ & $\mathrm{S} . \mathrm{S}$ & $\mathrm{L} . \mathrm{C}$ & $\mathrm{P} / \mathrm{Q}$ & $\mathrm{Sp}$ & $\mathrm{F} / \mathrm{Q}$ & $\mathrm{Sf}$ & $\mathrm{Q}_{\text {official }}$ \\
\hline Cairo-Embaba & 2 & 2.244 & single & electrical & 0 & 1.00 & 39 & 0.00 & 0 & 38 \\
\hline Embaba-Bashtel & 1 & 3.87 & single & electrical & 2 & 0.97 & 49 & 0.03 & 46 & 39 \\
\hline Bashtel-Wardan & 14 & 6.478 & single & electrical & 8 & 0.56 & 40 & 0.44 & 27 & 68 \\
\hline Wardan-Khatatba & 2 & 5.818 & single & electrical & 3 & 0.55 & 59 & 0.45 & 35 & 67 \\
\hline Khatatba-Tahrir & 5 & 5.555 & single & electrical & 5 & 0.55 & 48 & 0.45 & 32 & 66 \\
\hline Tahrir-Ethad & 4 & 4.924 & single & electrical & 2 & 0.55 & 44 & 0.45 & 33 & 66 \\
\hline Ethad-Etayelbarod & 9 & 4.297 & single & electrical & 9 & 0.88 & 39 & 0.12 & 30 & 41 \\
\hline Mamoura-Abokir & 2 & 1.991 & single & electrical & 0 & 1.00 & 33 & 0.00 & 0 & 112 \\
\hline Mamoura-Zahrya & 11 & 1.893 & single & electrical & 3 & 0.97 & 24 & 0.03 & 24 & 122 \\
\hline Zahrya-Alex & 3 & 2.79 & single & electrical & 8 & 1.00 & 32 & 0.00 & 0 & 118 \\
\hline Qaluib-Sheben & 7 & 4.081 & single & electrical & 13 & 0.55 & 33 & 0.45 & 27 & 58 \\
\hline Abozaabl-23yolyo & 7 & 2.071 & single & mechanical & 9 & 1.00 & 15 & 0.00 & 0 & 30 \\
\hline Abozaabl-Mahager & 2 & 1.559 & single & mechanical & 3 & 0.88 & 17 & 0.12 & 16 & 34 \\
\hline Mahager-Sheben & 5 & 2.233 & single & mechanical & 6 & 0.75 & 16 & 0.25 & 18 & 40 \\
\hline Banha-Menouf & 10 & 4.067 & single & mechanical & 16 & 1.00 & 31 & 0.00 & 0 & 24 \\
\hline Menouf-Kafrelzayat & 18 & 5.837 & single & mechanical & 25 & 1.00 & 32 & 0.00 & 0 & 20 \\
\hline Cairo-Kanater & 8 & 4.858 & single & mechanical & 15 & 0.90 & 35 & 0.10 & 35 & 42 \\
\hline Kanater-Ashmon & 7 & 5.88 & single & mechanical & 8 & 0.88 & 32 & 0.12 & 32 & 34 \\
\hline Ashmon-Menouf & 7 & 4.433 & single & mechanical & 16 & 0.89 & 34 & 0.11 & 31 & 36 \\
\hline
\end{tabular}




\begin{tabular}{|c|c|c|c|c|c|c|c|c|c|c|}
\hline Link Name & $\mathrm{Nb}$ & $\mathrm{Lb}$ & T.T & S.S & L.C & $P / Q$ & Sp & $\mathrm{F} / \mathrm{Q}$ & Sf & $Q_{\text {official }}$ \\
\hline Menouf-Tanta & 11 & 6.776 & single & mechanical & 12 & 0.89 & 37 & 0.11 & 29 & 36 \\
\hline Salmoum-Mahalfa & 13 & 23 & single & mechanical & 6 & 0.00 & 0 & 1.00 & 33 & 2 \\
\hline Mahalfa-Samala & 2 & 19 & single & mechanical & 2 & 0.50 & 31 & 0.50 & 26 & 4 \\
\hline Samala-Matroh & 1 & 11 & single & mechanical & 6 & 0.00 & 0 & 1.00 & 33 & 2 \\
\hline Rashed-Bosely & 3 & 7.3 & single & mechanical & 12 & 1.00 & 43 & 0.00 & 0 & 17 \\
\hline Bosely-Eltarh & 8 & 5.669 & single & mechanical & 13 & 0.73 & 35 & 0.27 & 33 & 22 \\
\hline Eltarh-Eltabya & 2 & 2.645 & single & mechanical & 0 & 0.67 & 36 & 0.33 & 35 & 24 \\
\hline Eltabya-Mamoura & 2 & 2.319 & single & mechanical & 0 & 0.83 & 29 & 0.17 & 25 & 24 \\
\hline Bosely-Metobus & 5 & 3.94 & single & mechanical & 10 & 0.83 & 31 & 0.17 & 30 & 46 \\
\hline Metobus-Desok & 8 & 5.4 & single & mechanical & 15 & 0.60 & 35 & 0.40 & 37 & 20 \\
\hline Metobus-Qasaby & 5 & 4.494 & single & mechanical & 7 & 1.00 & 30 & 0.00 & 0 & 8 \\
\hline Ethad-Kabary & 15 & 14.36 & single & mechanical & 26 & 0.00 & 0 & 1.00 & 32 & 24 \\
\hline Matroh-Eldabaa & 12 & 14.245 & single & mechanical & 21 & 0.71 & 68 & 0.29 & 36 & 21 \\
\hline Eldabaa-Hamam & 9 & 15.716 & single & mechanical & 8 & 0.73 & 60 & 0.27 & 36 & 22 \\
\hline Hamam-Gharbanyat & 1 & 10.926 & single & mechanical & 1 & 0.80 & 65 & 0.20 & 46 & 30 \\
\hline Gharbanyat-Borgelarab & 3 & 9.105 & single & mechanical & 2 & 0.71 & 52 & 0.29 & 40 & 34 \\
\hline Borgelarab-Qabary & 10 & 6.169 & single & mechanical & 19 & 0.72 & 49 & 0.28 & 38 & 39 \\
\hline Qabary-Moharambk & 3 & 1.552 & single & mechanical & 2 & 0.52 & 31 & 0.48 & 20 & 54 \\
\hline Moharambk-Arafa & 2 & 2.638 & single & mechanical & 1 & 0.43 & 36 & 0.57 & 28 & 46 \\
\hline Arafa-Nawatya & 2 & 1.121 & single & mechanical & 2 & 0.67 & 27 & 0.33 & 15 & 12 \\
\hline Nawatya-Sedygabr & 1 & 1.978 & single & mechanical & 1 & 1.00 & 26 & 0.00 & 0 & 7 \\
\hline Alex-Sedygabr & 2 & 2.793 & single & mechanical & 2 & 1.00 & 32 & 0.00 & 0 & 4 \\
\hline Ismailia-Nafesha & 3 & 1.68 & single & mechanical & 5 & 1.00 & 26 & 0.00 & 0 & 15 \\
\hline Nafesha-Fayed & 10 & 5.987 & single & mechanical & 13 & 0.33 & 42 & 0.67 & 39 & 57 \\
\hline Fayed-Geniva & 3 & 6.47 & single & mechanical & 6 & 0.31 & 43 & 0.69 & 44 & 55 \\
\hline Geniva-Swiss & 6 & 7.484 & single & mechanical & 7 & 0.32 & 48 & 0.68 & 41 & 56 \\
\hline Swiss-Ainshams & 17 & 17.08 & single & mechanical & 21 & 0.80 & 37 & 0.20 & 33 & 10 \\
\hline Swiss-Ainsokhna & 6 & 30.665 & single & mechanical & 6 & 0.00 & 0 & 1.00 & 21 & 16 \\
\hline Zagazig-Sheben & 13 & 5.979 & single & mechanical & 21 & 0.54 & 39 & 0.46 & 30 & 48 \\
\hline Zagazig-Abokbir & 8 & 5.889 & single & mechanical & 16 & 0.66 & 44 & 0.34 & 36 & 47 \\
\hline Abokbir-Mansoura & 16 & 4.938 & single & mechanical & 11 & 0.59 & 38 & 0.41 & 33 & 39 \\
\hline Mansoura-Dakahlya & 25 & 5.16 & single & mechanical & 31 & 1.00 & 33 & 0.00 & 0 & 16 \\
\hline Fakous-Abokir & 5 & 5.638 & single & mechanical & 9 & 1.00 & 35 & 0.00 & 0 & 22 \\
\hline Fakous-Salhya & 6 & 5.699 & single & mechanical & 10 & 1.00 & 35 & 0.00 & 0 & 20 \\
\hline Fakous-Samaana & 5 & 2.224 & single & mechanical & 4 & 1.00 & 30 & 0.00 & 0 & 10 \\
\hline Mansoura-Domyat & 22 & 5.728 & single & mechanical & 40 & 0.54 & 37 & 0.46 & 25 & 50 \\
\hline Sherben-Beyla & 8 & 5.39 & single & mechanical & 13 & 0.87 & 38 & 0.13 & 36 & 30 \\
\hline Beyla-Kafrelsheikh & 9 & 8.5 & single & mechanical & 25 & 0.88 & 39 & 0.12 & 34 & 34 \\
\hline Kafrelsheikh-Qelin & 8 & 2.83 & single & mechanical & 9 & 0.89 & 32 & 0.11 & 33 & 38 \\
\hline Tanta-Mahaletroh & 6 & 4.14 & single & mechanical & 7 & 0.79 & 46 & 0.21 & 37 & 38 \\
\hline Mahaletroh-Qelin & 10 & 4.837 & single & mechanical & 14 & 0.71 & 37 & 0.29 & 27 & 42 \\
\hline Qelin-Desok & 6 & 4.268 & single & mechanical & 13 & 0.76 & 37 & 0.24 & 35 & 34 \\
\hline Desok-Damanhour & 9 & 3.401 & single & mechanical & 9 & 1.00 & 33 & 0.00 & 0 & 26 \\
\hline
\end{tabular}




\begin{tabular}{|c|c|c|c|c|c|c|c|c|c|c|}
\hline Link Name & $\mathrm{Nb}$ & $\mathrm{Lb}$ & T.T & S.S & L.C & $P / Q$ & $\mathrm{Sp}$ & $\mathrm{F} / \mathrm{Q}$ & Sf & $Q_{\text {official }}$ \\
\hline Tanta-Santa & 3 & 5.362 & single & mechanical & 9 & 0.84 & 44 & 0.16 & 45 & 32 \\
\hline Santa-Metghamr & 6 & 4.914 & single & mechanical & 7 & 0.76 & 35 & 0.24 & 33 & 34 \\
\hline Metghamr-Zagazig & 13 & 3.475 & single & mechanical & 21 & 0.75 & 32 & 0.25 & 32 & 32 \\
\hline Santa-Mahaletroh & 6 & 5.225 & single & mechanical & 8 & 0.91 & 37 & 0.09 & 35 & 23 \\
\hline Banha-Metghamr & 14 & 4.281 & single & mechanical & 16 & 1.00 & 32 & 0.00 & 0 & 22 \\
\hline Teben-Marazik & 2 & 3.215 & single & mechanical & 2 & 0.09 & 32 & 0.91 & 14 & 22 \\
\hline Marazik-Hawamdeya & 3 & 4.723 & single & mechanical & 4 & 0.09 & 54 & 0.91 & 39 & 22 \\
\hline Hawamdeya-Bolak & 6 & 5.503 & single & mechanical & 6 & 0.08 & 42 & 0.92 & 36 & 24 \\
\hline Bolak-Cairo & 3 & 6.441 & single & mechanical & 2 & 0.20 & 49 & 0.80 & 28 & 10 \\
\hline Wasta-Adwaa & 6 & 7 & single & mechanical & 8 & 0.76 & 47 & 0.24 & 31 & 33 \\
\hline Adwaa-Fayom & 2 & 5 & single & mechanical & 4 & 0.81 & 45 & 0.19 & 35 & 31 \\
\hline Cairo-Qaluib & 4 & 4.858 & double & electrical & 3 & 0.91 & 54 & 0.09 & 36 & 111 \\
\hline Qaluib-Banha & 6 & 7.451 & double & electrical & 14 & 0.84 & 91 & 0.16 & 45 & 120 \\
\hline Banha-Tanta & 10 & 5.918 & double & electrical & 20 & 0.75 & 72 & 0.25 & 43 & 134 \\
\hline Tanta-Kafrelzayat & 4 & 6.598 & double & electrical & 7 & 0.76 & 78 & 0.24 & 41 & 131 \\
\hline Kafrelzayat-Etayelbarod & 6 & 4.955 & double & electrical & 5 & 0.78 & 73 & 0.22 & 42 & 128 \\
\hline Damanhour-Etayelbarod & 5 & 5.903 & double & electrical & 6 & 0.81 & 91 & 0.19 & 47 & 128 \\
\hline Damnhour-Abis & 10 & 6.434 & double & electrical & 18 & 0.82 & 73 & 0.18 & 45 & 132 \\
\hline Alex-Abis & 5 & 2.793 & double & electrical & 1 & 0.96 & 31 & 0.04 & 115 & 104 \\
\hline Cairo-Bolak & 3 & 6.441 & double & electrical & 1 & 0.85 & 39 & 0.15 & 26 & 89 \\
\hline Bolak-Giza & 1 & 3.231 & double & electrical & 1 & 0.78 & 39 & 0.22 & 29 & 97 \\
\hline Giza-Marazik & 8 & 5.503 & double & electrical & 9 & 0.81 & 68 & 0.19 & 41 & 109 \\
\hline Marazik-Wasta & 13 & 5.746 & double & electrical & 33 & 0.79 & 85 & 0.21 & 40 & 111 \\
\hline Wasta-Minya & 33 & 8.646 & double & electrical & 61 & 0.82 & 69 & 0.18 & 35 & 103 \\
\hline Minya-Asuit & 29 & 7.033 & double & electrical & 39 & 0.83 & 65 & 0.17 & 42 & 99 \\
\hline Asuit-Sohag & 21 & 6.674 & double & electrical & 31 & 0.80 & 64 & 0.20 & 46 & 87 \\
\hline Sohag-Nagahamady & 19 & 6.872 & double & electrical & 25 & 0.76 & 58 & 0.24 & 40 & 74 \\
\hline Nagahamady-Qena & 13 & 7.222 & double & electrical & 16 & 0.76 & 61 & 0.24 & 42 & 74 \\
\hline Qena-Auxsor & 14 & 6.526 & double & electrical & 0 & 0.75 & 61 & 0.25 & 40 & 72 \\
\hline Auxsor-Edfo & 24 & 7.847 & double & electrical & 29 & 0.71 & 60 & 0.29 & 41 & 62 \\
\hline Edfo-Aswan & 27 & 6.72 & double & electrical & 32 & 0.71 & 57 & 0.29 & 41 & 62 \\
\hline Aswan-Sadali & 4 & 12.788 & double & electrical & 5 & 1.00 & 38 & 0.00 & 0 & 13 \\
\hline Qaluib-Zagazig & 6 & 7.451 & double & mechanical & 10 & 0.72 & 83 & 0.28 & 48 & 46 \\
\hline Banha-Zagazig & 14 & 4.657 & double & mechanical & 19 & 0.64 & 50 & 0.36 & 22 & 76 \\
\hline Zagazig-Baawla & 8 & 7.348 & double & mechanical & 19 & 0.52 & 51 & 0.48 & 42 & 92 \\
\hline Baawla-Nafesha & 7 & 7.683 & double & mechanical & 10 & 0.53 & 61 & 0.47 & 42 & 90 \\
\hline Nafesha-Ismailia & 3 & 1.68 & double & mechanical & 5 & 0.68 & 32 & 0.32 & 20 & 63 \\
\hline Ismailia-Raswa & 10 & 11.062 & double & mechanical & 12 & 0.55 & 59 & 0.45 & 33 & 44 \\
\hline Raswa-Portsaid & 1 & 5.212 & double & mechanical & 3 & 1.00 & 51 & 0.00 & 0 & 24 \\
\hline Tanta-Mahaletroh & 6 & 4.3 & double & mechanical & 7 & 0.78 & 52 & 0.22 & 41 & 67 \\
\hline Mahaletroh-Mahala & 5 & 3.538 & double & mechanical & 12 & 0.77 & 47 & 0.23 & 44 & 74 \\
\hline Mahala-Mansoura & 11 & 5.004 & double & mechanical & 26 & 0.75 & 39 & 0.25 & 38 & 68 \\
\hline
\end{tabular}




\section{RESULTS \& ANALYSIS}

Applying this procedure on the Egyptian railway network which consists of 104 links classified into double and single links with either mechanical or electrical signal system. There are three types of links according to the number of passenger and freight trains running on them: links for passenger and freight trains (P/F), only for passenger trains $(P)$ and only for freight trains $(F)$.

The correlation matrix was obtained between the input and output data as shown in table 2 .

The mathematical models of capacity calculation can be determined using the combination of the factors based on their importance obtained from neural network model which can be expressed by track type (T.T), signal system (S.S), the longest block (Lb), passenger speed (Sp) and freight speed (Sf) [15] by SPSS under the normal operation system. The models fulfilled the factors sign and the significance of model coefficients. In addition, the coefficient of determination $\left(R^{2}\right)$ was calculated in order to investigate variation and nonlinearity between the inputs and output data and validate the used model.

Model 1: For links of passenger/freight trains:

$\mathrm{Q}=22.151+23.187 \mathrm{~T} . \mathrm{T}+35.034 \mathrm{~S} . \mathrm{S}-1.485 \mathrm{Lb}+$

$+0.218 \mathrm{Sp}+0.354 \mathrm{Sf}$

$\mathrm{R}^{2}=0.717$, Absolute error $=19.195$
Model 2: For links of passenger trains:

$\mathrm{Q}=25.362+25.356 \mathrm{~T} . \mathrm{T}+35.429 \mathrm{~S} . \mathrm{S}-$

$$
-1.252 \mathrm{Lb}+0.33 \mathrm{Sp}
$$

$R^{2}=0.694$, Absolute error $=19.863$

Model 3: For links of freight trains:

$$
\mathrm{Q}=28.878+26.960 \mathrm{~T} . \mathrm{T}+36.436 \mathrm{~S} \text {.S- }
$$

$-1.557 \mathrm{Lb}+0.406 \mathrm{Sf}$

$R^{2}=0.711$, Absolute error $=19.32$

The three above models were used to calculate the optimum factors values. These equations were consisted of numeric factors and symbols. The symbols were considered as dummy variables for track types and signal systems. In equations, replaced the double track by 1 and single track by 0 Also replaced electrical signal by 1 and mechanical signal by 0 . The optimum factors were calculated to determine the maximum railway capacity applying iteration technique by solver program as shown in table 3.

Table 2: Correlation matrix between the input factors and the official capacity $\left(Q_{\text {official }}\right)$

\begin{tabular}{|c|c|c|c|c|c|c|c|c|c|c|}
\hline Affecting Factors & $\mathrm{Nb}$ & $\mathrm{Lb}$ & $\mathrm{T} . \mathrm{T}$ & $\mathrm{S} . \mathrm{S}$ & $\mathrm{L.C}$ & $\mathrm{P} / \mathrm{Q}$ & $\mathrm{Sp}$ & $\mathrm{F} / \mathrm{Q}$ & $\mathrm{Sf}$ & $\mathrm{Q}_{\text {official }}$ \\
\hline $\mathrm{Nb}$ & 1 & & & & & & & & & \\
\hline $\mathrm{Lb}$ & 0.1938 & 1 & & & & & & & & \\
\hline $\mathrm{T} . \mathrm{T}$ & 0.2612 & 0.0157 & 1 & & & & & & & \\
\hline S.S & 0.1971 & -0.0810 & 0.5220 & 1 & & & & & & \\
\hline L.C & 0.8735 & 0.1486 & 0.2624 & 0.0958 & 1 & & & & & \\
\hline $\mathrm{P} / \mathrm{Q}$ & 0.0198 & -0.4517 & 0.1204 & 0.1935 & 0.0457 & 1 & & & & \\
\hline Sp & 0.1953 & -0.0534 & 0.6185 & 0.4610 & 0.2496 & 0.1653 & 1 & & & \\
\hline F/Q & -0.0198 & 0.4517 & -0.1204 & -0.1935 & -0.0457 & -1 & -0.1653 & 1 & & \\
\hline Sf & 0.1263 & 0.1518 & 0.3808 & 0.2511 & 0.1194 & -0.2821 & 0.4294 & 0.2821 & 1 & \\
\hline$Q_{\text {official }}$ & 0.1707 & -0.1964 & 0.6756 & 0.7294 & 0.1819 & 0.1331 & 0.5961 & -0.1331 & 0.4240 & 1 \\
\hline
\end{tabular}


Table 3: The optimal effective factors for maximize railway capacity

\begin{tabular}{|c|c|c|c|c|c|}
\hline Link types & Traffic Composition & Capacity Equation & Conditions & Factors Range & $\begin{array}{c}\text { Optimum } \\
\text { Factor }\end{array}$ \\
\hline \multirow{2}{*}{$\begin{array}{l}\text { Double- } \\
\text { electrical }\end{array}$} & \multirow{2}{*}{$\begin{array}{c}\mathrm{P} / \mathrm{F} \\
(20 \text { links) }\end{array}$} & \multirow{2}{*}{$\begin{array}{c}Q=80.372- \\
1.485 L b+0.218 S p+0.354 S f\end{array}$} & $S p=53.206+0.225 S f$ & \multirow{2}{*}{$\begin{array}{c}\mathrm{Lb}=2.79: 12.78 \\
\mathrm{Sp}=31: 91 \\
\mathrm{Sf}=26: 46\end{array}$} & \multirow{2}{*}{$\begin{array}{c}\mathrm{Lb}=6 \\
\mathrm{Sp}=64 \\
\mathrm{Sf}=46\end{array}$} \\
\hline & & & $S p=54.568-0.33 L b+0.239 S f$ & & \\
\hline \multirow{2}{*}{$\begin{array}{l}\text { Double- } \\
\text { mechanical }\end{array}$} & \multirow{2}{*}{$\begin{array}{c}\mathrm{P} / \mathrm{F} \\
\text { (9links) }\end{array}$} & \multirow{2}{*}{$\begin{array}{c}Q=45.338- \\
1.485 L b+0.218 S p+0.354 S f\end{array}$} & $S p=46.357+0.225 S f$ & \multirow{2}{*}{$\begin{array}{c}\mathrm{Lb}=1.68: 11.06 \\
\mathrm{Sp}=39: 83 \\
\mathrm{Sf}=20: 48\end{array}$} & \multirow{2}{*}{$\begin{array}{l}\mathrm{Lb}=7.4 \\
\mathrm{Sp}=57 \\
\mathrm{Sf}=48\end{array}$} \\
\hline & & & $S p=48.14-0.33 L b+0.239 S f$ & & \\
\hline \multirow{3}{*}{$\begin{array}{l}\text { Single- } \\
\text { electrical }\end{array}$} & \multirow[b]{2}{*}{$\begin{array}{c}\mathrm{P} / \mathrm{F} \\
(8 \mathrm{links})\end{array}$} & \multirow[b]{2}{*}{$\begin{array}{c}Q=57.185- \\
1.485 \mathrm{Lb}+0.218 S p+0.354 S f\end{array}$} & $S p=36.274+0.221 S f$ & \multirow{2}{*}{$\begin{array}{c}\mathrm{Lb}=1.893: 6.478 \\
\mathrm{Sp}=24: 59 \\
\mathrm{Sf}=0: 46\end{array}$} & \multirow{2}{*}{$\begin{array}{l}\mathrm{Lb}=6.1 \\
\mathrm{Sp}=47 \\
\mathrm{Sf}=46\end{array}$} \\
\hline & & & $S p=35.635+0.212 S f+0.172 L$ & & \\
\hline & $P($ (3links) & $Q=60.791-1.252 L b+0.33 S p$ & $S p=42.95-0.187 L b$ & $\begin{array}{c}\mathrm{Lb}=1.99: 2.79 \\
\mathrm{Sp}=32: 43\end{array}$ & $\begin{array}{c}\mathrm{Lb}=1.99 \\
\mathrm{Sp}=42\end{array}$ \\
\hline \multirow{4}{*}{$\begin{array}{l}\text { Single- } \\
\text { mechanical }\end{array}$} & \multirow{2}{*}{$\begin{array}{c}\mathrm{P} / \mathrm{F} \\
(44 \text { links) }\end{array}$} & \multirow{2}{*}{$\begin{array}{c}Q=22.15- \\
1.485 L b+0.218 S p+0.354 S f\end{array}$} & $S p=30.841-0.33 L b+0.239 S f$ & \multirow{2}{*}{$\begin{array}{c}\mathrm{Lb}=1.12: 17.08 \\
\mathrm{Sp}=16: 68 \\
\mathrm{Sf}=14: 46\end{array}$} & \multirow{2}{*}{$\begin{array}{l}\mathrm{Lb}=7.2 \\
\mathrm{Sp}=40 \\
\mathrm{Sf}=46\end{array}$} \\
\hline & & & $S p=29.118+0.225 S f$ & & \\
\hline & $P($ 14links) & $Q=25.362-1.252 L b+0.33 S p$ & $S p=35.708-0.187 \mathrm{Lb}$ & $\begin{array}{c}\mathrm{Lb}=1.68: 7.3 \\
\mathrm{~S} p=15: 43\end{array}$ & $\begin{array}{c}\mathrm{Lb}=1.68 \\
\mathrm{Sp}=35\end{array}$ \\
\hline & $\mathrm{F}$ (4links) & $Q=28.878-1.557 L b+0.406 S f$ & $S f=20.340+0.6 \mathrm{Lb}$ & $\begin{array}{l}\mathrm{Lb}=11: 20 \\
\mathrm{Sf}=21: 33\end{array}$ & $\begin{array}{l}\mathrm{Lb}=11 \\
\mathrm{Sf}=27\end{array}$ \\
\hline
\end{tabular}

\section{CONCLUSIONS}

This paper presents an analytical model which can be used by railway agencies to improve railway practical capacity. The practical capacity can be useful for long term strategic capacity planning.

The analysis performed in this paper determine railway capacity during normal operation and improved the reliability of capacity analysis in future taking into consideration the most effective factors that affect on capacity calculation are the longest block section (Lb), passenger speed (Sp) and freight speed (Sf).

Applying the proposed methodology on Egyptian national railway (ENR):

For passenger and freight links, the practical capacity will be maximized when:

- The optimum value of factors will be: $L b=7.4 \mathrm{~km}$, $\mathrm{Sp}=57 \mathrm{kph}$ and $\mathrm{Sf}=48 \mathrm{kph}$ for double-mechanical links. The capacity of 6links will be increased.

- The optimum value of factors will be: $L b=6 \mathrm{~km}$, $\mathrm{Sp}=64 \mathrm{kph}$ and $\mathrm{Sf}=46 \mathrm{kph}$ for double-electrical links. The capacity of 13 links will be increased.

- The optimum value of factors will be: $L b=7.2 \mathrm{~km}$, $\mathrm{Sp}=40 \mathrm{kph}$ and $\mathrm{Sf}=46 \mathrm{kph}$ for single-mechanical links. The capacity of 37 links will be increased.

- The optimum value of factors will be: $L b=5.9 \mathrm{~km}$, $\mathrm{Sp}=47 \mathrm{kph}$ and $\mathrm{Sf}=46 \mathrm{kph}$ for single-electrical links. The capacity of 7 links will be increased.

For passenger links only, the practical capacity will be maximized when:
- The optimum value of factors will be: $L b=1.68 \mathrm{~km}$ and $\mathrm{Sp}=35 \mathrm{kph}$ for single-mechanical links. The capacity of 14 links will be increased.

- The optimum value of factors will be: $L b=1.99 \mathrm{~km}$ and $\mathrm{Sp}=43 \mathrm{kph}$ for single-electrical links. The capacity of 3 links will be increased.

For freight links only, the practical capacity will be maximized when:

- The optimum value of factors will be: $L b=11 \mathrm{~km}$ and $\mathrm{Sf}=27 \mathrm{kph}$ for single-mechanical links. The capacity of 3links will be increased.

The line capacity of more than $80 \%$ of the Egyptian railway network can be increased under some changes in block length, passenger and freight speeds without changing the track type (single or double), signal system (electrical or mechanical) and traffic composition.

For double electrical and double mechanical links, there are only one link for passenger trains in ENR. Applying the equation to calculate the practical capacity, the capacity can be increased more than 40 to $60 \%$ of official capacity. That means the links are incompletely maximum used.

To realize the optimum block length, two approaches should be followed:

- The block section lengths more than the optimum divide into two approximately equal by constructing a new refuse siding (additional block section).

In double links, No need to construct sidings at all block section except at through stations to receive the freight trains on refuge siding, while in single links, it is proposed 
to construct sidings at all block section ass they are considered not only refuge siding but also confluence of two opposite trains.

- The sum of successive block section length proposes to be divided by the optimum length to obtain the suitable number of blocks and reconstructed this part of link to maximize the line capacity.

To improve the average speed for obtaining the optimum speed, three scenarios will be proposed:

1. Decrease the train composition by reducing number of cars per train which decrease the revenue

2. Use locomotives types having horsepower more than the actual one

3. Examine the average speed between two successive block sections and modify the speed which less than the optimum speed for both passenger and freight trains by decreasing the stop points during their trip.

In such a way, the first train is proposed to stop at the first station but it pass by the second one,etc while the second train will pass by the first station and stop at the second one( reciprocal movement). Then the official time table will be rearranged to obtain the speed equal or more than the optimum speed. This alternative will save cost and time.

\section{REFERENCES}

1. Robots, C., Schmid, F., Connor, P., Ramads, V., and Sharpe, J. (2010). A New Railway System Capacity Model, A Method of Assessing Capability Tradeoffs on Railway Capacity Using A System-Wide Approach. Transport Research Laboratory.

2. International Union of Railway Capacity. UIC code 405, 406. $1^{\text {st }}$ edition 1996. And, $2^{\text {nd }}$ edition 2004.

3. HDR Engineering. (2001). Everett to Blaine Commuter Rail Preliminary Feasibility Study. Washington: Report prepared for the Washington State Department of Transportation and Snohomish County.

4. Lindfeldt, A., (2015). Railway Capacity Analysis, "Methods for Simulation and Evaluation of Timetables, Delays and Infrastructure". PhD Thesis in Infrastructure Stockholm, KTH Royal Institute of Technology.

5. Pachl, J., (2018). Railway Operation and Control, 4th Edition, VTD Rail Publishing, WA, United States.

6. Abril, M., Barber, F., Ingolotti, L., Salido, M. A., Tormos, P., Lova, A., (2008). An Assessment of Railway Capacity, Transportation Research Part E: Logistics and Transportation Review, vol. 44 (5), pp. 774-806. DOI:10.1016/j.tre.2007.04.001.

7. Han, J., Yue, Y., Zhou L., (2016). A Microscopic Simulation Method to Calculate the Capacity of Railway, Station School of Traffic \& Transportation, China International Forum on Management, Education and Information Technology Application.
8. Ali, M.A., (2012). Opportunities for High-Speed Railways in Developing and Emerging Countries: A case study Egypt. PhD Thesis of the Railways Engineering Approved Dissertation. DOI: 10.14279/depositonce-3343.

9. Connor, P., (2014). High Speed Railway Capacity Understanding the factors affecting capacity limits for a high speed railway.

10. Huelsz, A.D. Prince, (2015). Capacity Factors in Intermodal Road Rail Terminals. Department of Technology Management and Economics. Division of Logistics and Transportation. DOI: 10.13140/ RG.2.1.4945.5127.

11. Sangphong, O., Siridhara, S., and Ratanavaraha V., (2016). Determining Critical Rail Line Blocks and Minimum Train Headways for Equal and Unequal Block Lengths and Various Train Speed Scenarios. DOI: 10.4186/ej.2017.21.3.281.

12. Zhong, Q., Shaoquan Ni, Shengdong Li, Chang'an Xu., (2019). Railway Infrastructure Capacity Utilization Description through Data Integration in Blocking Time Theory, $8^{\text {th }}$ International Conference on Railway Operations Modelling and Analysis - Rail Norrkoping.

13. Perugachi-Diaz, Y., (2017). Correlation in Linear Regression, Vrije Universiteit Amsterdam Research Paper.

14. Kim M., Bruce A. Mccarl, and Thomas H. Spreen. (2013). Applied Mathematical Programming.

15. Diana, M.S. Rahoma, Ali Z. Heikal, Haytham N. Zohny, Akram S. Kotb. (2019). Parametric Model for Evaluating Railway Network Capacity Using Neural Network Techniques, International Journal of Engineering and Advanced Technology (IJEAT) SSN: 2249 - 8958, Volume-X, Issue-X.

Paper submitted: 05.08.2020.

Paper accepted: 05.10.2020.

This is an open access article distributed under the CC BY 4.0 terms and conditions. 